\title{
Algorithm of Blind Pixels Detection for IRFPA Based on Integration Time Adjustment
}

\author{
Shaosheng DAI ${ }^{1}$, Yongqiang $\mathrm{LIU}^{2}$, Zhihui $\mathrm{DU}^{3}$ and Fei $\mathrm{XU}^{4}$
}

\begin{abstract}
In order to effectively detect blind pixels for Infrared Focal Plane Array (IRFPA), this paper provides a new algorithm of blind pixels detection. The algorithm realizes blind pixels detection by distinguishing responsivity between effective pixels and ineffective pixels in different integration time during the process of IRFPA real-time imaging. The experimental results show that the algorithm can effectively locate blind pixels without the help of black body. It is simple operation and has a broad application prospect in engineering.
\end{abstract}

Keywords: IRFPA; Blind pixel detection; Integration time adjustment

\section{Introduction}

The application field of infrared imaging system has been broadened with the rapid development of the IRFPA Technology. However, nonuniformity of response is widespread between the each detection unit in IRFPA owing to being limited by materials and processing technology [1]. Some detection units have no response or response slowly and forms non-effective pixels when the external thermal radiation changes. The non-effective pixel is also called blind pixel. The distribution and the number of blind pixels have a great influence on device performance. Without any processing, a large number of white spots or black spots will emerge in the output image of the infrared imaging system, so there are too many blind pixels. Therefore, detection and compensation of blind pixels can improve the performance of infrared imaging system.

The paper analyses the feature of blind pixels and proposes a new algorithm of blind pixels detection based on integration time adjustment. It can be applied to real-time blind pixels detection and has better effect than the traditional methods.

\footnotetext{
${ }^{3}$ Zhihui DU $(\square)$

Chongqing Key Laboratory of Signal and Information Processing, Chongqing University of Posts and Telecommunications, Chongqing, China

e-mail: eliteduzhihui@outlook.com
} 


\section{Definition of blind pixel}

Following are the definition of relevant parameters of blind pixel [2]:

\subsection{Pixel responsivity}

Assume there is a size $\alpha+\beta=x$ IRFPA with certain frame frequency and dynamic range, the output signal voltage of the pixel $(i, j)(i \in[1, M], j \in[1, N])$ under per unit irradiation power is referred to as pixel responsivity $R(i, j)$, shown as equation (1):

$$
R(i, j)=V_{s}(i, j) / P
$$

In the equation, $V_{s}(i, j)$ represents responding voltage of pixel $(i, j)$ to irradiation power $P, P$ represents the irradiation power on pixel $(i, j)$.

\subsection{Average responsivity of detector}

Average responsivity represents arithmetic average of effective pixels' responsivity on IRFPA, shown as equation (2):

$$
\bar{R}=\frac{1}{M \cdot N-(d+h)} \sum_{i=1}^{M} \sum_{j=1}^{N} R(i, j)
$$

In this equation, $d$ and $h$ represents number of dead pixels and over-heating pixels respectively.

\subsection{Blind pixel}

Blind pixel is also known as non-effective pixel, which includes dead pixels and over-heating pixels. According to Chinese national standard [3], over-heating pixel is defined as pixel with responsivity tenfold larger than average, dead pixel is defined as pixel with responsivity one-tenth less than the average.

\subsection{Blind pixel rate}

Percentage of non-effective pixels on IRFPA, shown as equation (3): 


$$
N_{b}=\frac{d+h}{M \cdot N} \times 100 \%
$$

\section{Blind Pixel Detection Based on Integration Time Adjustment}

\subsection{Traditional blind pixel detection based on blackbody}

Common blind pixel detection methods [4] are shown as follow:

- Detection based on definition. Set tenfold and one-tenth of grayscale average responsivity of all pixels in entire frame as the threshold of blind pixel detection, and judge each pixel's gray value by the tenfold. This method is easy to implement, but has the disadvantage of high chance of mistaken judgment.

- Double reference-source principle. Use two blackbody radiation sources with different temperature to irradiate IRFPA, thus difference between detector's responding to two blackbodies and difference between average responsivity to two blackbodies can be measured. If the difference is tenfold larger or onetenth less than the average responsivity gray value, this pixel is defined as blind pixel. In another way, blind pixel can be judged through comparison between pixels' responsivity difference and preset threshold. In actual application, threshold is calculated through multiple iterations based on pixels' responsivity to two radiation sources and the threshold of blind pixels' definition, and the exact number of blind pixels is required to calculate the threshold. Obviously double reference-source principle cannot real-timely detect random blind pixels during the processing of using IRFPA.

- Threshold principle. Divide area under blackbody's irradiation into upper and lower parts and preset a comparison threshold, then iterate through the upper part, calculate gray value difference between each pixel and its following five and downside five pixels, if the difference is larger than threshold, then judge the pixel as blind pixel. And iterate lower part similarly. Algorithm of this method has advantage of less calculation amount. But time and temperature drift would influence the accuracy of detection. Thus the method would require blackbody irradiation to acquire responsivity difference and need to stop imaging process.

- $3 \sigma$ principle. Theoretical model of blind pixel: under the irradiation of uniform blackbody with temperature $T$, responsivity of detector and noise distribution are obeying normal distribution: $\varphi(x)=(2 \pi \sigma)^{-\frac{1}{2}} \exp \left[-(x-\mu)^{2} / 2 \sigma^{2}\right]$, in which $\mu$ represents expected gray value of detector, $\sigma$ represents mean- 
square deviation of gray value. Under the condition that the temperature is fixed, calculate one frame's expected gray value $\mu$ and noise voltage's meansquare deviation $\sigma$.If pixel's response value exceed $\mu \pm 3 \sigma$, Then the pixel can be judged as blind pixel.

- Combination of above methods, or preprocess blind pixel before applying these methods.

\subsection{Integration Time's Influence on Dynamic Range}

Integration time refers to the time of IRFPA detector's pixel accumulating irradiation signal and charging. As for IRFPA, many performance indicators are relevant to integration time, such as system's output voltage, responsivity, noise and specific detectivity. Selecting proper integration time has crucial influence on system's overall performance, especially system's output response. First, this paper theoretically analyzes integration time's influence on IRFPA system's output response, which shows that output response is proportional to integration time. Then demonstrate the conclusion through experiment, and thus prove that it is crucial to select different integration time to match corresponding irradiation intensity.

Literature [5] has pointed out that system's output response is proportional to integration time when IRFPA detector's circuit parameters are certain. Experiments show that by adjusting integration time, response value in different integration time can be obtained. Literature [6] has pointed out that response characteristics of blind pixel and effective pixel are different. Effective pixel is linear in certain dynamic range, and blind pixel's dynamic range is away from the effective pixel's. Characteristic curve of blind pixel's temperature response is generally nonlinear, and slope of curve is often lower or higher than normal, so difference of responses to two different temperatures is different to effective pixel's. Similarly, blind pixel and effective pixel show different response characteristic in different integration time. Through above analysis, adjusting integration time may influence IRFPA's response output, which provides theoretical foundation for blind pixel detection.

\subsection{Procedure of Blind Pixel Detection}

1. Record detector unit's response output values in different integration time.

- Record response output values in different integration time. According to the range of integration time, by adjusting step length of integration time, from the minimum value $T_{\max }$, sequentially superpose $\Delta T$ until value reaches $T_{\max }$, thus 
acquire IRFPA's response output values in different integration time, record and save them;

- Modeling IRFPA detector unit response value (shown as graph 1). Graph the characteristics curve of IRFPA's response output value as integration time changes.

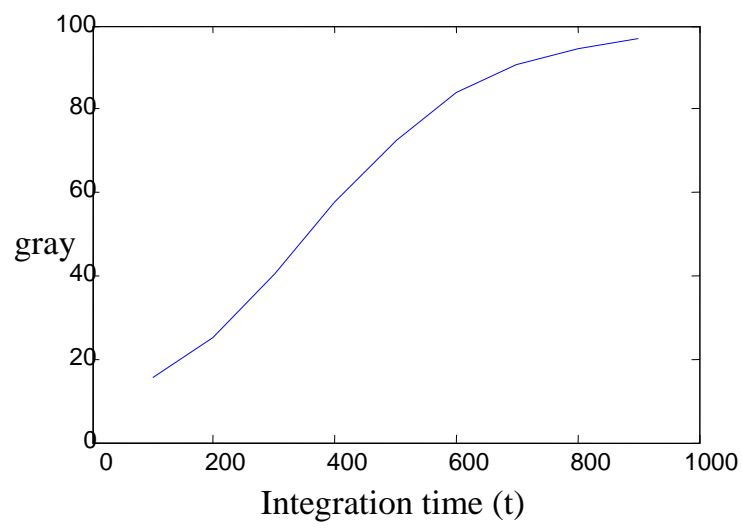

Fig. 1 IRFPA detector unit response model

2. Blind pixel detection. This method detects blind pixel by adjusting integration time. Assume the size of IRFPA is $M \times N$. First, cover the lens using lens cap, then acquire continuous $k$ pixels' gray value in linear interval of IRFPA response model, calculate their arithmetic average as pixels' responsivity, expressed as $R_{i j}$ in equation, shown as equation (4):

$$
R_{i j}=\frac{\sum_{n=0}^{k} X_{i j}\left(T_{l i n}+n \Delta T\right)}{k+1}
$$

In this equation, $i=1,2, \cdots, M$ and $j=1,2, \cdots, N, T_{\text {lin }}$ represents the start of integration time, $\Delta T$ represents the step length of integration time, $X_{i j}\left(T_{\text {lin }}+n \Delta T\right)$ represents pixel $(i, j)$ 'response gray value when the integration time is $T_{\text {lin }}+n \Delta T$. Procedure of Blind Pixel Detection is as follow:

- Search the gray values of pixels in window $(2 h+1) \times(2 h+1)$ at a certain integration time, and find their maximum and minimum gray value $R_{\max } 、 R_{\min }$.

- Remove $R_{\max } 、 R_{\min }$, and calculate remaining pixels' gray value arithmetic average $\bar{R}$, shown as equation (5): 


$$
\bar{R}=\frac{\sum_{i=h}^{i+h} \sum_{j=h}^{j+h} R_{i j}-R_{\max }-R_{\min }}{(2 h+1) \times(2 h+1)-2}
$$

- Compare $R_{\max } 、 R_{\min }$ and $\bar{R}$. According to Chinese national standard GB/Tl744421998,"The technical norms for measurement and test of characteristic parameters of IRFPA", a pixel can be judged as blind pixel when $\bar{R}>10 R_{\max }$ or $\bar{R}<10 R_{\min }$, record the location of blind pixels and mark them in corresponding location in image matrix.

\section{Experimental Results}

The experiment adopts 320×240 Pyroelectric Infrared Focal Plane Array imaging system with blind pixels. Record the grey scale images in the different integration time under the constant temperature circumstance and calculate the grey scale of each image, as the Figure 2 shows. Take $3 \times 3$ window size of blind pixels detection as an example. The experimental results are shown in the Figure 3.
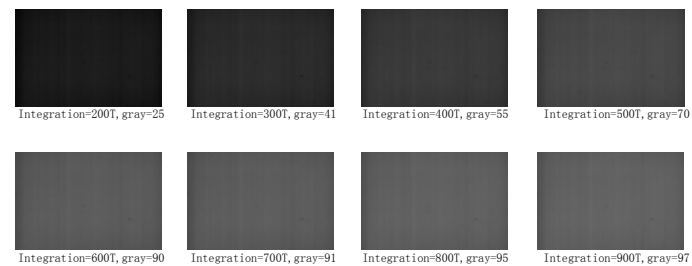

Fig. 2 Grey scale images in different integration time

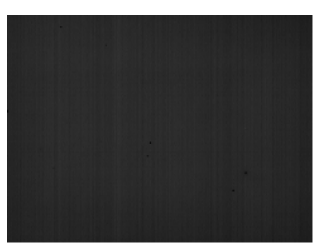

(a) original image

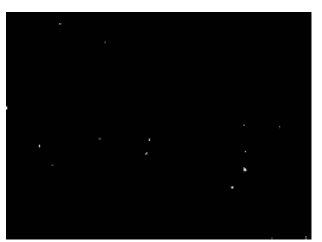

(b) detecting image

Fig.3 Blind pixels detection results

This paper provides a new algorithm of blind pixels detection. The algorithm realizes blind pixels accurate detection by distinguishing responsivity between effective pixels and blind pixels on different integration time during IRFPA real-time imaging process. The algorithm effectively detects blind pixels in IRFPA without the help of usually standard black body radiation sources. Since it can detect blind pixels by turning on the switch anytime and anywhere which is simple to operate, 
its dominant feature indicates the broad application prospects in the practical project.

\section{Acknowledgements}

This work is supported by the National Natural Science Foundation of China (No.61275099, No.61102131), the Project of Key Laboratory of Signal and Information Processing of Chongqing (No.CSTC2009CA2003), the Natural Science Foundation of Chongqing Science and Technology Commission (No.CSTC2010BB2411, CSTC2010BB2398, CSTC2006BB2373), the Natural Science Foundation of Chongqing Municipal Education Commission (No.KJ060509, KJ080517), the Science and technique foundation of Chongqing (CSTC, 2011AB2008), and the Natural Science Foundation of Chongqing University of Posts and Telecommunications (CQUPT) (A2006-04, A2006-86).

\section{References}

1. Chen R, Tan XQ (2002). Study on Non-uniformity Correction of Infrared Image. Infrared Technology, 24(1), 1-3.

2. Li YJ, Cui RQ, Zhao GF and Yuan QG (2007). A New Method of Bad Pixel Detection and Compensation of IRFPA. Laser \& Infrared, 37(1), 51-52, 65.

3. GB 17444-1998. The technical norms for measurement and test of characteristic parameters of infrared focal plane arrays.

4. Zhang XN (2010). Improved blind element inspection algorithm of infrared FPA array. Laser \& Infrared, 40(10), 1149-1153.

5. Li FW, Zhang YQ (2008). Influence of integration time on IRFPA imaging system. Applied Optics, 29(5), 727-730.

6. Zhou HX, Yin SM, Liu SQ and Lai R (2004). Algorithm of Blind Pixels Auto-searching and Compensation for IRFPA. 33(5), 598-600. 